\title{
Effets des intempéries sur les portails romans : les cas de Montceaux-l'Étoile (Saône-et-Loire) et Chassenard (Allier)
}

En hommage à François Voinchet, architecte en chef des Monuments historiques, décédé brutalement en hiver 2007, sur la route des églises romanes.

\section{Juliette Rollier-Hanselmann}

\section{(2) OpenEdition}

12 Journals

\section{Édition électronique}

URL : https://journals.openedition.org/cem/1128

DOI : $10.4000 /$ cem. 1128

ISSN : 1954-3093

Éditeur

Centre d'études médiévales Saint-Germain d'Auxerre

\section{Édition imprimée}

Date de publication : 15 août 2007

ISSN : 1623-5770

Référence électronique

Juliette Rollier-Hanselmann, «Effets des intempéries sur les portails romans : les cas de Montceauxl'Étoile (Saône-et-Loire) et Chassenard (Allier) », Bulletin du centre d'études médiévales d'Auxerre I BUCEMA [En ligne], 11 | 2007, mis en ligne le 29 septembre 2011, consulté le 22 septembre 2022. URL : http://journals.openedition.org/cem/1128; DOI : https://doi.org/10.4000/cem.1128

Ce document a été généré automatiquement le 22 septembre 2022.

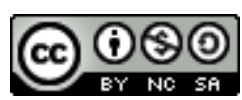

Creative Commons - Attribution - Pas d'Utilisation Commerciale - Partage dans les Mêmes Conditions 4.0 International - CC BY-NC-SA 4.0

https://creativecommons.org/licenses/by-nc-sa/4.0/ 


\section{Effets des intempéries sur les portails romans : les cas de Montceaux-l'Étoile (Saône-et-Loire) et Chassenard (Allier)}

En hommage à François Voinchet, architecte en chef des Monuments historiques, décédé brutalement en hiver 2007, sur la route des églises romanes.

Juliette Rollier-Hanselmann

1 Les portails romans de Bourgogne, bien que connus de tous les spécialistes, n'ont jamais fait l'objet d'un constat d'état ou bilan sanitaire.

2 Le tympan unique de Montceaux-l'Étoile a subi de nombreuses exfoliations et son épiderme est maintenant très érodé. Le portail sud d'Anzy-le-Duc perd régulièrement des morceaux sculptés. Un cavalier, le visage d'Ève et d'autres fragments ont été recollés récemment, tandis que la jambe d'Adam a disparu durant les dernières décennies.

3 Le portail récemment découvert à Chassenard était protégé des intempéries et conserve une statuaire exceptionnellement bien préservée.

4 Je vous propose d'établir un premier bilan comparatif entre deux ensembles sculptés situés dans la même aire géographique, l'un conservé à l'extérieur, l'autre à l'abri des intempéries.

\section{Montceaux-I'Étoile}


Fig. 1 - Montceaux-l'Étoile, vue du portail (cl. J. Rollier).

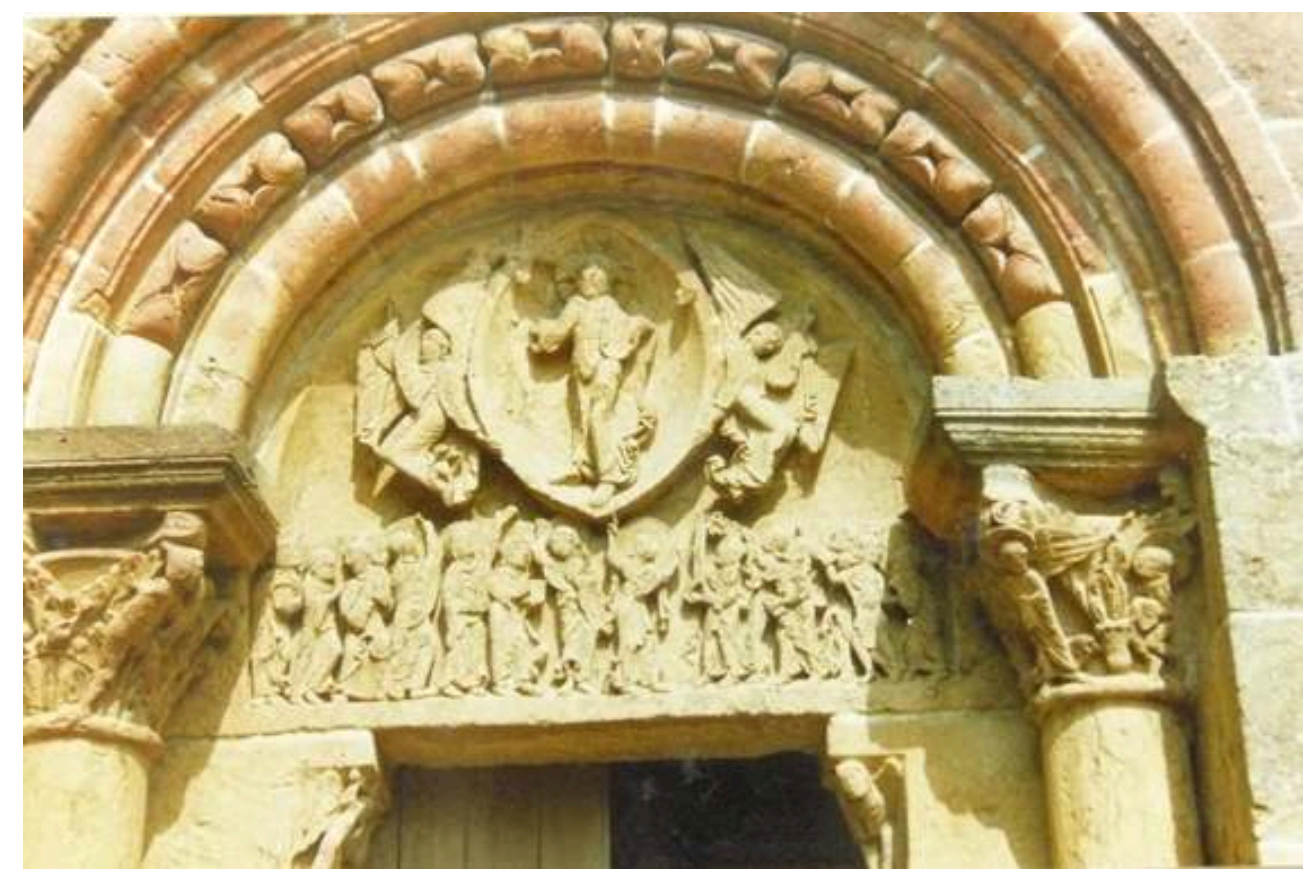

En 1967, l'historien d'art Y. Christe ${ }^{1}$ avait relevé des relations iconographiques entre l'Ascension sculptée d'un type rare et certains ivoires carolingiens. Plus récemment la thèse de $\mathrm{W}$. Travis ${ }^{2}$ a enrichi nos connaissances sur ce portail, mais nous n'entrerons pas dans le détail de l'étude iconographique ici. Le constat qu'il fait à partir d'une photographie de $1938^{3}$ indique que divers fragments de sculpture sont tombés (morceaux du Christ et un fragment du bras supérieur du diable situé sur la console nord).

6 Notre comparaison avec les photographies prises par les éditions Zodiaque en 1979 montrent que les reliefs très travaillés des bases supportant les colonnes latérales ont presque complètement disparu ${ }^{4}$.

7 Il semblerait que le portail ait été protégé des intempéries pendant le XIX ${ }^{\mathrm{e}}$ siècle et au début du XX $X^{\mathrm{e}}$ siècle, comme l'atteste J. Virey en 1926: « la façade fut débarassée d'un porche sans style qui l'avait longtemps masquée ». La photographie qu'il publie montre une construction maçonnée sur les côtés.

8 Les pierres rougies de la façade indiquent que le feu a ravagé le porche, à une époque indéterminée. Deux encoches obliques situées à la hauteur des contreforts marquent l'emplacement de la structure qui protégeait autrefois les sculptures.

9 Suite à une étude préalable de l'architecte F. Didier en 1994, des travaux de restauration extérieure ont été réalisés en deux tranches annuelles, le chœur et le clocher en 1996, la nef et le draînage périphérique en $1997{ }^{5}$. Devant la porte ouest un nouveau dallage a été posé. La façade a été reprise, avec le changement d'un certain nombre de pierres grises, qui ont subi un traitement de vieillissement par flammage, ce qui a permis de retrouver la teinte des pierres rubéfiées encore en place, et d'harmoniser ainsi les parties neuves et les parties anciennes ${ }^{6}$. Un schéma des interventions prévues se trouve dans l'étude préalable, mais le dossier signale que les remplacements ont été plus nombreux que prévu. En 1997 une petite intervention de 
nettoyage du portail a été effectuée (préconsolidation au silicate d'éthyle, microsablage) ${ }^{7}$.

10 L'observation rapprochée sur échafaudage ${ }^{8}$ permet de constater l'état très usé des sculptures. La plupart des apôtres sont défigurés par l'érosion et les desquamations. La tête de saint Pierre ne conserve plus qu'un petit monticule à la place du nez et les yeux ont quasiment disparu. En revanche dans les parties protégées de la pluie, les sculptures sont en bien meilleur état, comme par exemple le petit ange situé à droite sous le linteau, personnage qui conserve encore ses yeux (la pupille est marquée au trépan) ${ }^{9}$.

Fig. 2 - Montceaux-l'Étoile, détail de l'érosion de la pierre (cl. J. Rollier).

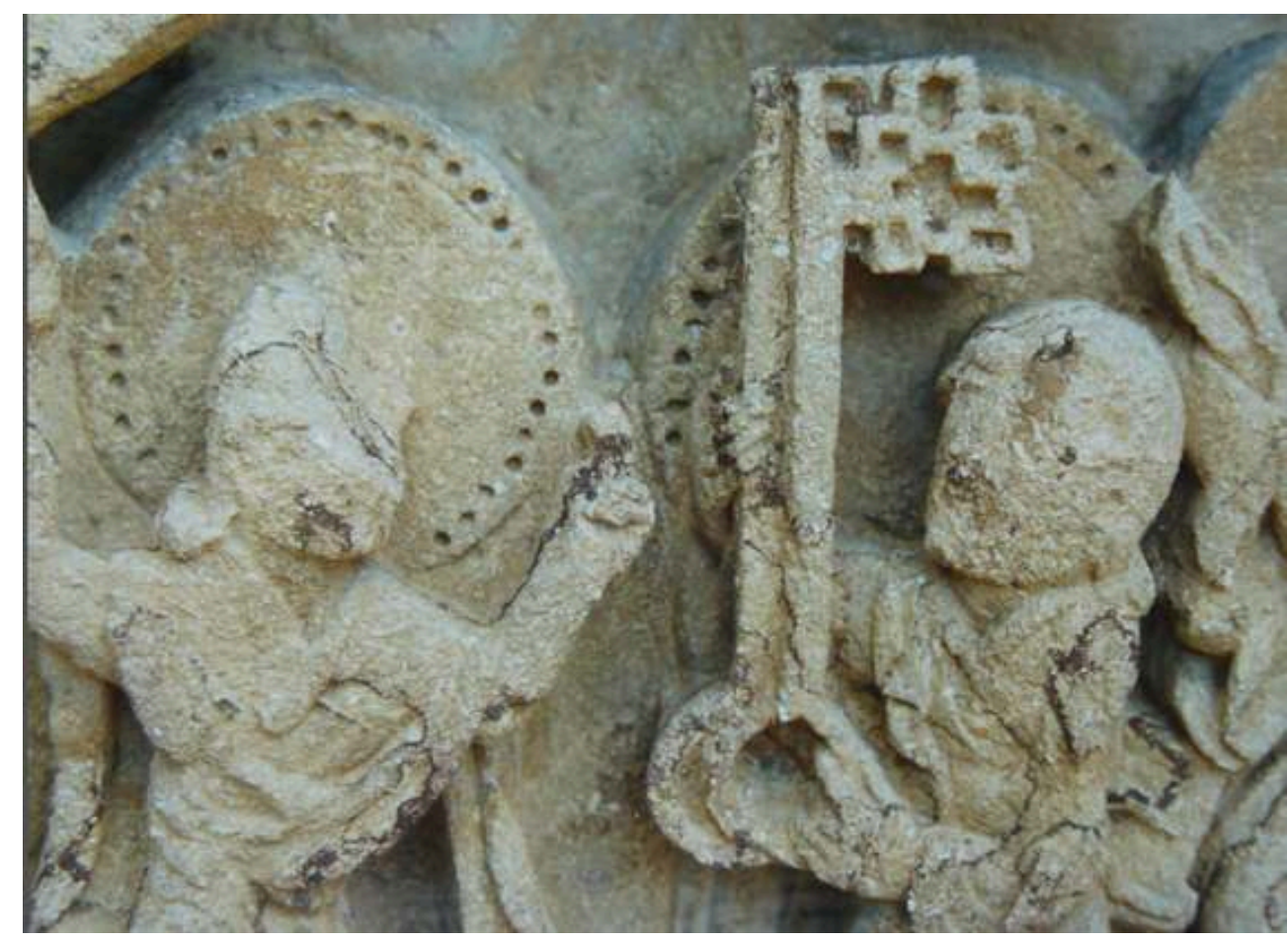

11 La dégradation des sculptures peut être due à plusieurs facteurs. Tout d'abord le support utilisé - un calcaire à entroque contenant de nombreux petits coquillages- qui est un matériau tendre et poreux. Cette belle pierre jaune a permis la réalisation d'une sculpture finement travaillée, dont l'aspect de surface original est encore bien visible dans les recoins protégés. Par contre sa structure irrégulière et sa porosité la rendent très fragile lorsqu'elle est directement exposée aux intempéries, comme c'est le cas ici. 
Fig. 3 - Montceaux-l'Étoile, détail de l'érosion de la pierre (cl. J. Rollier).

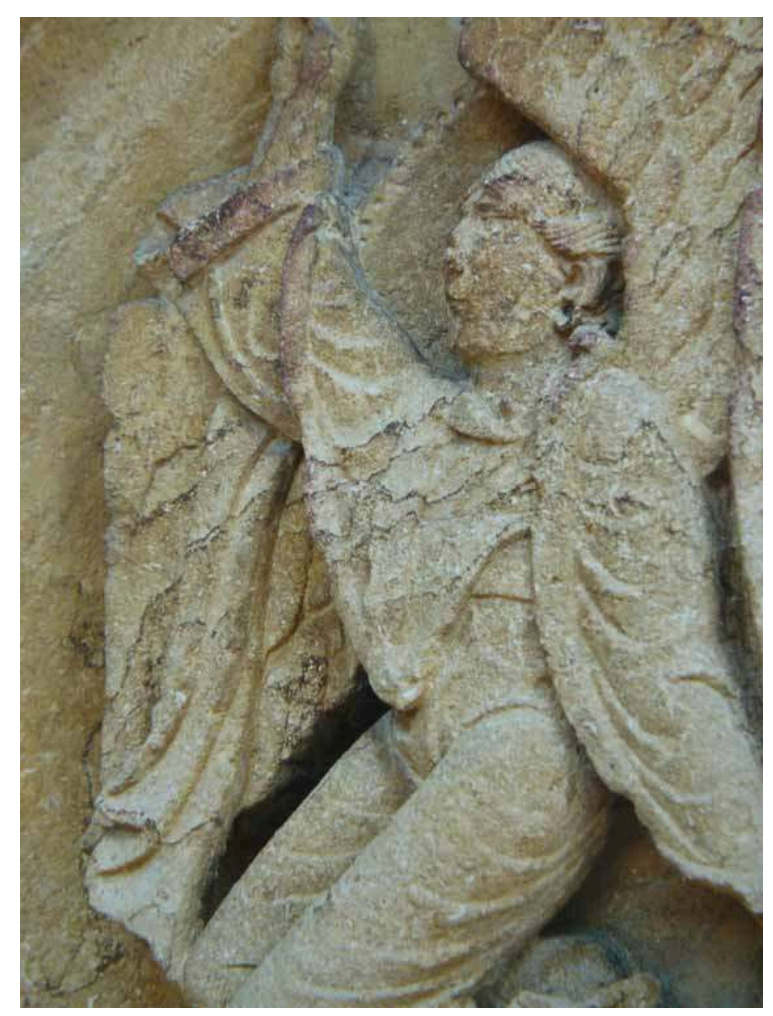

12 Ce petit portail n'est malheureusement que très peu protégé par les quelques voussures qui le surmontent, si bien que l'agression du vent et de la pluie l'usent très rapidement, sans parler des effets du gel. Au point de vue iconographique, il s'agit d'un type très rare de représentation de l'Ascension conservé actuellement en France, si bien que tout devrait être entrepris pour sa préservation.

13 La remise en place du porche ne paraît cependant pas être une opération très coûteuse, ni compliquée. Elle permettrait de protéger des eaux pluviales, et dans une certaine mesure de l'érosion éolienne.

\section{Chassenard}

14 Un portail exceptionnellement bien conservé fut découvert fortuitement en octobre 2000 à l'église paroissiale de Chassenard, située à quelques kilomètres d'Anzy-le-Duc. Une première publication a été faite par Annie Regond ${ }^{10}$, conservateur des Antiquités et Objets d'art, qui demanda le classement immédiat du portail. 
Fig. 4 - Chassenard, vue du portail lors de la découverte (J. Rollier).

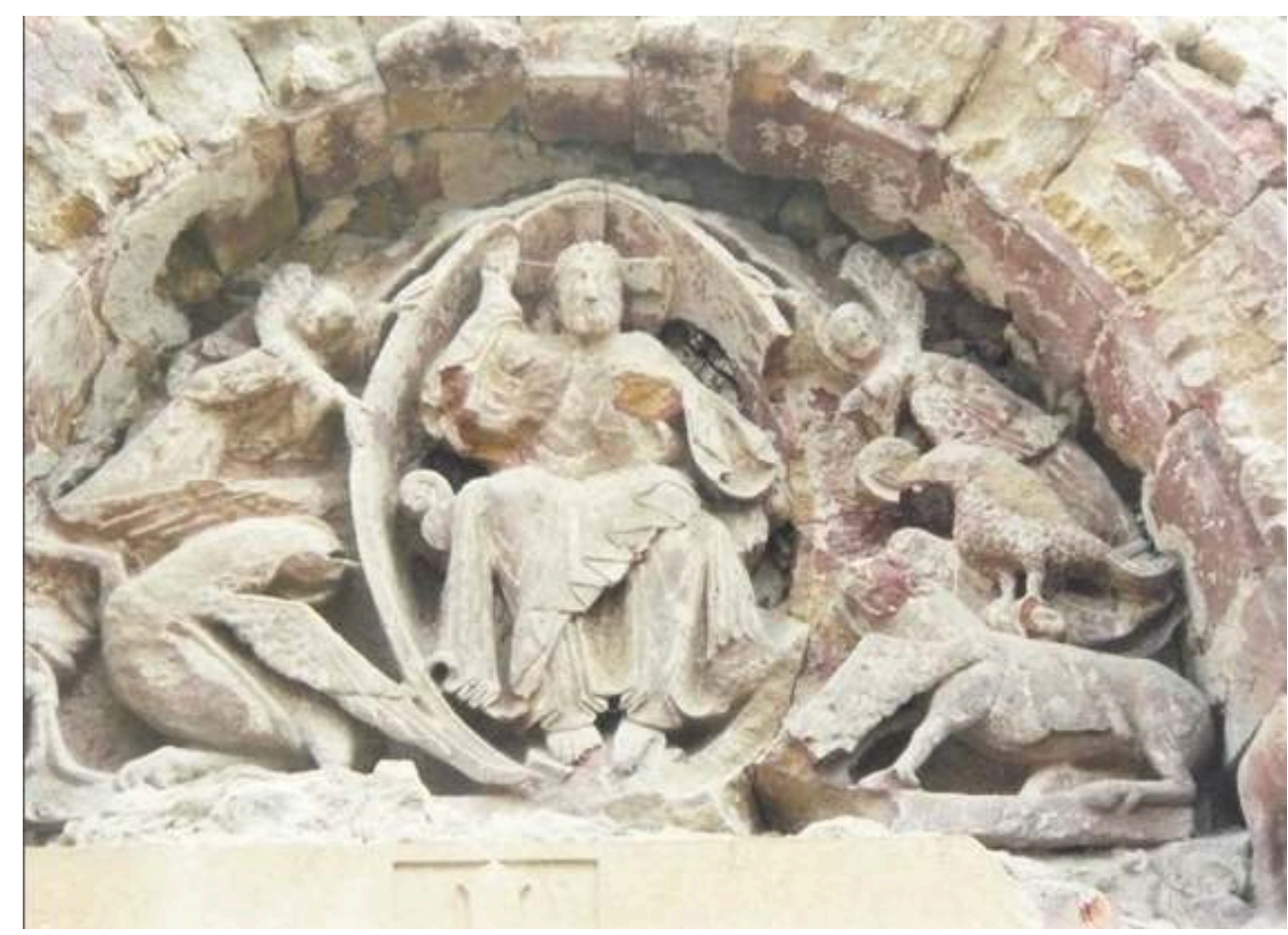

Quelques mois après la découverte, le portail fut préservé des intempéries par un châssis en bois et un double voile d'intissé polyester ${ }^{11}$. Le portail étant complètement déstabilisé (déversement vers l'extérieur, fissures diverses), une structure en bois a été mise en place afin de prévenir des éventuels mouvements ${ }^{12}$.

Le tympan est occupé par le Christ en Majesté, assis sur un siège à arcature, qui bénit de la main droite. Il est entouré des symboles des évangélistes. Le linteau, en partie détruit par la mise en place d'une porte au XIX ${ }^{e}$ siècle, conserve encore des fragments de personnages. Suite à la découverte de la frise en 1939-1945, N. Stratford ${ }^{13}$ établissait déjà une relation étroite avec les sculptures de Neuilly-en-Donjon (tympan) et de SaintLéger-sur-Vouzance (chapiteaux du chœur). L'étude du portail permet de confirmer ces comparaisons et de situer l'ensemble vers 1140 . Un relevé archéologique permet également de connaître les difficultés de mise en place de cette statuaire, mais ceci est un autre sujet.

L'épiderme de la pierre est exceptionnellement bien conservé (aspect très lisse, détails sculptés préservés), excepté pour l'extrados qui présente des altérations anciennes dues au ruissellement des eaux pluviales. Les marques d'un incendie ont provoqué un rougissement de la pierre calcaire, avec éclatement d'un certains nombre de fragments, notamment les éléments en haut-relief.

18 De nombreux restes d'enduits et de badigeons subsistent. Sur les éléments figurés, nous constatons la présence d'une couche picturale de facture très simple. La tête du Christ conserve divers restes de couleurs : lèvres rouges, fragment de sourcil gris, pommettes roses, front et nez roses. Le contour externe de l'oreille est marqué en rose. L'encolure $\mathrm{du}$ vêtement $\mathrm{du}$ Christ est perceptible, bien que fortement pâlie par le temps: la tunique est blanche, avec le contour de l'encolure marqué en noir et des plis rouges. Il semblerait que le peintre a simplement cherché à faire ressortir les creux et les reliefs. 

capteur d'humidité-température ${ }^{15}$. Les mesures enregistrées indiquent de très fortes variations d'humidité entre le jour et la nuit (jusqu'à $35 \%$ d'H.R. entre le jour et la nuit), informations qui devront être prises en compte pour la conservation des restes polychromes et la mise en place du porche. Durant l'hiver et le printemps, les taux d'humidité relative sont hauts, avoisinant souvent les 85-90\% d'H.R. (humidité relative). De façon générale, on assiste à des variations brutales de température et d'humidité relative suivant le cycle jour/nuit et, ce, tout au long de l'année. Ces variations n'ont que peu d'effet sur la pierre qui est un matériau relativement inerte en terme de dilatation thermique. En revanche, cela peut être plus ennuyeux pour les matériaux annexes, tels que les couches polychromes, plus ou moins sensibles à ces écarts selon les techniques picturales. Les périodes de gel constatées en hiver ne sont pas anodines, d'autant plus que les taux d'humidité relative durant cette période sont assez élevés.

26

nge, à gauche, qui soutient la mandorle conserve également de nombreux restes de badigeon blanc, teinté de rose sur les mains et le visage (pommettes). contours gris subsistent autour d'un œil. couleurs sont généralement très pulvérulents. portail de Senlis (1165-1170) et le jubé de Vezzolano (Piémont, daté du début XIII ${ }^{e}$ siècle). La première polychromie de Senlis montre des personnages en vêtement blanc, bordé d'or. La mise en teinte vise essentiellement à faire ressortir les principaux éléments pour les rendre plus lisibles de loin. Le jubé de Vezzolano conserve également des colorations relativement simples. Le Christ porte une tunique blanche, dont l'encolure est marquée d'un simple filet noir. Par contre, un large manteau bleu de lapis-lazuli l'entoure également.

(cheveux des anges «noyés » dans le badigeon), mais donnent un aspect « velouté » aux carnations. Dans l'hypothèse d'un incendie ancien, qui aurait faire disparaître les couleurs originales, il faut imaginer une campagne de rénovation dont la date pose évidemment problème. Même s'il s'agit d'une restauration récente, il paraît important de conserver toutes les informations contenues par ce seul ensemble.

un avant-toit en matériau mixte (tôle ondulée en métal et plastique). Le portail reste cependant fortement exposé à l'échauffement solaire (façade sud). La mise en place d'un porche proposée par l'architecte en chef F. Voinchet, est en cours d'étude. L'expérience de la structure provisoire permet de se rendre compte que le porche définitif devra être suffisamment élevé et éloigné du sommet du tympan pour tempérer l'effet du soleil. Un simple auvent tel qu'on l'observe à Saint-Pierre-le-Moutier (Nièvre) ${ }^{14}$ s'avère en effet inefficace, les parties inférieures étant balayées par les pluies obliques.

Les conditions climatiques du lieu sont connues, grâce aux données enregistrées par un En conclusion, le constat que nous sommes amenés à faire à propos des portails exposés aux intempéries (Anzy-le-Duc, Montceaux-l'Étoile) est alarmant, dans la mesure où le rythme des dégradations est très rapide depuis la couverture photographique des 
années 1970. L'état désastreux du portail sud du cloître d'Anzy-le-Duc et l'usure avancée de celui de Montceaux-l'Étoile, en comparaison avec l'épiderme quasi intact des tympans protégés (portails du musée du Hiéron et de Chassenard), devraient stimuler rapidement la réflexion sur la protection de la statuaire monumentale exposée aux intempéries. Le restaurateur se doit d'alerter les acteurs du patrimoine et la communauté scientifique au sujet de ce patrimoine en péril. L'acharnement thérapeutique ne suffit pas à contenir les dégâts dus aux intempéries. Il est maintenant nécessaire de revoir notre mode d'intervention et de préconiser la mise en place très rapide de porches de protection.

INDEX

Index géographique : France/Montceaux l'Étoile, France/Chassenard

Mots-clés : portail roman 